\title{
Diabetes induces dysregulation of microRNAs associated with survival, proliferation and self-renewal in cardiac progenitor cells
}

\author{
Nima Purvis ${ }^{1}$ - Sweta Kumari ${ }^{1}$. Dhananjie Chandrasekera ${ }^{1}$. Jayanthi Bellae Papannarao ${ }^{1} \cdot$ Sophie Gandhi ${ }^{1}$. \\ Isabelle van Hout ${ }^{1} \cdot$ Sean Coffey ${ }^{2} \cdot$ Richard Bunton $^{3} \cdot$ Ramanen Sugunesegran $^{3}$ - Dominic Parry ${ }^{3} \cdot$ Philip Davis $^{3}$. \\ Michael J. A. Williams ${ }^{2} \cdot$ Andrew Bahn ${ }^{1} \cdot$ Rajesh Katare $^{1}$
}

Received: 5 October 2020 / Accepted: 15 December 2020 / Published online: 2 March 2021

(C) The Author(s), under exclusive licence to Springer-Verlag GmbH, DE part of Springer Nature 2021

\begin{abstract}
Aims/hypothesis Diabetes mellitus causes a progressive loss of functional efficacy in stem cells, including cardiac progenitor cells (CPCs). The underlying molecular mechanism is still not known. MicroRNAs (miRNAs) are small, non-coding RNA molecules that regulate genes at the post-transcriptional level. We aimed to determine if diabetes mellitus induces dysregulation of miRNAs in CPCs and to test if in vitro therapeutic modulation of miRNAs would improve the functions of diabetic CPCs. Methods CPCs were isolated from a mouse model of type 2 diabetes $(d b / d b)$, non-diabetic mice and human right atrial appendage heart tissue. Total RNA isolated from mouse CPCs was miRNA profiled using Nanostring analysis. Bioinformatic analysis was employed to predict the functional effects of altered miRNAs. MS analysis was applied to determine the targets, which were confirmed by western blot analysis. Finally, to assess the beneficial effects of therapeutic modulation of miRNAs in vitro and in vivo, prosurvival miR-30c-5p was overexpressed in mouse and human diabetic CPCs, and the functional consequences were determined by measuring the level of apoptotic cell death, cardiac function and mitochondrial membrane potential (MMP).

Results Among 599 miRNAs analysed in mouse CPCs via Nanostring analysis, 16 miRNAs showed significant dysregulation in the diabetic CPCs. Using bioinformatics tools and quantitative real-time PCR (qPCR) validation, four altered miRNAs (miR30c-5p, miR-329-3p, miR-376c-3p and miR-495-3p) were identified to play an important role in cell proliferation and survival. Diabetes mellitus significantly downregulated miR-30c-5p, while it upregulated miR-329-3p, miR-376c-3p and miR-495-3p. MS analysis revealed proapoptotic voltage-dependent anion-selective channel 1 (VDAC1) as a direct target for miR-30c-5p, and cell cycle regulator, cyclin-dependent protein kinase 6 (CDK6), as the direct target for miR-329-3p, miR-376c-3p and miR-4953 p. Western blot analyses showed a marked increase in VDAC1 expression, while CDK6 expression was downregulated in diabetic CPCs. Finally, in vitro and in vivo overexpression of miR-30c-5p markedly reduced the apoptotic cell death and preserved MMP in diabetic CPCs via inhibition of VDAC1.

Conclusions/interpretation Our results demonstrate that diabetes mellitus induces a marked dysregulation of miRNAs associated with stem cell survival, proliferation and differentiation, and that therapeutic overexpression of prosurvival miR-30c-5p reduced diabetes-induced cell death and loss of MMP in CPCs via the newly identified target for miR-30c-5p, VDAC1.
\end{abstract}

Keywords Cardiac progenitor cells $\cdot$ Diabetes mellitus $\cdot$ microRNA $\cdot$ miR-30c-5p $\cdot$ Mitochondrial membrane potential Stem cell therapy

Andrew Bahn and Rajesh Katare contributed equally to the study.

Rajesh Katare

rajesh.katare@otago.ac.nz

Andrew Bahn

andrew.bahn@otago.ac.nz

1 Department of Physiology-HeartOtago, School of Biomedical Sciences, University of Otago, Dunedin, New Zealand
2 Department of Medicine, Dunedin School of Medicine, University of Otago, Dunedin, New Zealand

3 Department of Cardiothoracic Surgery, Dunedin School of Medicine, University of Otago, Dunedin, New Zealand 


\section{Research in context}

\section{What is already known about this subject?}

- Diabetes induces progressive loss of cardiovascular cells, including cardiac progenitor cells

- Studies have identified the impaired therapeutic potential of diabetic stem cells. However, the underlying mechanism for this impairment is not known

- MicroRNAs play a crucial role in the pathophysiological process of several diseases

\section{What is the key question?}

- Does diabetes-induced dysregulation of microRNAs underpin the impaired potential of cardiac progenitor cells?

\section{What are the new findings?}

- Diabetes induced significant dysregulation in the microRNAs that are associated with survival, proliferation and self-renewal in mouse and human cardiac progenitor cells

- The functional implication of dysregulated miRNAs was confirmed by altered target proteins

- In vitro and in vivo overexpression of the downregulated prosurvival miR-30c markedly reduced death and preserved mitochondrial membrane potential in diabetic cardiac progenitor cells

How might this impact on clinical practice in the foreseeable future?

- Therapeutic modification of microRNAs could be an effective approach in improving the therapeutic potential of diabetic cardiac progenitor cells for the treatment of patients following an ischaemic insult

\section{Abbreviations}

A.U. Arbitrary units

CPC Cardiac progenitor cell

CDK6 Cyclin-dependent kinase 6

GSK-3 $\beta \quad$ Glycogen synthase kinase 3 beta

MEF2C Myocyte-specific enhancer factor 2C

miRNA microRNA

MMP Mitochondrial membrane potential

PTP Permeability transition pore

qPCR Quantitative real-time PCR

RAA Right atrial appendage

Sca- $1^{+} \quad$ Stem cell antigen-1 positive

3'UTR 3'-Untranslated region

VDAC1 Voltage-dependent anion-selective channel 1

\section{Introduction}

Cardiovascular disease is currently the leading cause of death in the world for both men and women and is responsible for more than 17 million deaths each year [1]. Although current pharmacotherapy slows down heart failure progression and improves survival prognosis, the only curative treatment, besides heart transplantation, would be the replacement of lost myocytes by new ones, the ultimate goal of regenerative cardiology [2-4]. Over the past two decades, various stem cells have been tested to regenerate lost cardiomyocytes. The results were mixed with some being positive and some being negative, including recent controversy on the specific type of stem cells [5-7]. Despite this recent uncertainty, stem cell therapy is still an attractive therapeutic approach for the treatment of cardiovascular disease [8], owing to the multifactorial property of stem cells. Stem cells secrete paracrine factors that not only have angiogenic and cell survival properties, but also attract endogenous stem cells [9, 10]. Interestingly, the majority of studies have identified the impaired therapeutic potential of stem cells, including cardiac progenitor cells (CPCs), collected from individuals with diabetes or animal models of diabetes [11-13].

Several mechanisms have been implicated in the pathophysiology of stem cells from diabetic individuals or animals, such as impaired insulin signalling, increased oxidative stress, mitochondrial dysfunction and abnormal epigenetic alterations [3]. However, the precise molecular mechanisms underlying the impaired efficacy of diabetic stem cells are still not known. We, along with others, have shown a marked dysregulation of microRNAs (miRNAs) starting at the early stage in the diabetic heart [14-16]. miRNAs are small (21-25 nucleotide), non-coding RNA molecules that regulate gene expression at the post-transcriptional level. Recently, we demonstrated that dysregulation of miRNAs from an early stage in the diabetic heart forms a foundation for the development of functional and structural abnormalities at a later stage [17-19]. Evidence from previous studies has supported the crucial role of miRNAs in stem cell survival, proliferation and differentiation $[3,20]$. However, almost nothing is known about the effect of diabetes mellitus on miRNAs in CPCs. In 
this study, we investigated the effects of diabetes on the expression of miRNAs and determined the consequences for target proteins involved in survival and proliferation of CPCs from a mouse model of type 2 diabetes and from human right atrial appendage (RAA) heart tissue. We hypothesised that diabetes will induce dysregulation of miRNAs associated with stem cell survival, proliferation and differentiation and that therapeutic modulation of these miRNAs will improve the survival of both human and mouse diabetic CPCs.

\section{Methods}

Detailed methodology is provided in the electronic supplementary material (ESM) Methods.

Ethics Collection of human RAA tissue has been approved by the Health and Disability Ethics Committee of New Zealand (Healthy Volunteers Ethics Reference: Upper South A Ethics Committee Approval No. 01/05/062). All volunteers provided written consent for the tissue collection and use of samples for this study. Collection and use of the human samples were also in concordance with the Declaration of Helsinki. All experiments using mouse tissue have been approved by the University of Otago animal ethics committee (D25/12 and AUP 18-205).

Isolation of mouse and human CPCs Stem cell antigen-1 positive $\left(\mathrm{Sca}-1^{+}\right)$mouse CPCs were isolated from $10 \pm 1$ week-old male diabetic ( $d b / d b\left[\right.$ BKS.Cg-Dock $7 m^{+/+}$Leprdb/ $J$ ) and age-matched ( \pm 1 -week) non-diabetic mice as described previously [11]. Animals were bred at the Hercus Taieri Resource Centre of the University of Otago. Obese $d b /$ $d b$ mice (together with their non-diabetic lean littermates as controls) used as a model of type 2 diabetes best represent the human form of type 2 diabetes, and have been demonstrated to be a good model to study diabetes-induced cardiovascular disease $[15,17,18,21,22]$. All the animals used in this study were housed at $21 \pm 1{ }^{\circ} \mathrm{C}$ with food and water ad libitum.

Human CPCs were isolated from RAA biopsies collected from diabetic and age-matched ( \pm 3 years) non-diabetic patients ( $n=6$ each, ESM Table 1), undergoing on-pump coronary artery bypass surgery, after written and verbal consent, as described by us previously $[11,23]$. The experimenters were not blinded to the type of cells used in the study.

Morphologically, the Sca- $1^{+}$mouse CPCs exhibited a similar shape, but also a greater cross-sectional area after 1 week of culture (ESM Fig. 1a-c). The purity of the resulting cells was confirmed by flow cytometry analysis for the expression of specific markers and immunofluorescence analysis to exclude contamination by fibroblasts (ESM Results and ESM Fig. 1d, e) [11]. The cells were further expanded for the downstream applications.
miRNA expression profiling using Nanostring microarray and bioinformatics analysis Total RNA was extracted from diabetic $(n=6)$ and non-diabetic mouse CPCs $(n=5)$ using the QIAGEN miRNeasy Mini kit (Qiagen, USA) as per the manufacturer's instructions $[18,24]$ to perform the microarray analyses as described previously [25]. miRNAs were selected based on significant fold change $(p<0.05)$ in the diabetic CPCs.

The miRPath bioinformatics tool (microT-CDS, version 5, DIANA TOOLS) [26] was used to categorise the differentially regulated miRNAs according to their known function in stem cells. Four separate prediction databases (microT-CDS, version 5 [26], miRDB, version 3 [27], microRNA.org, version 1 [28] and TargetScan, version 6.2 [29]) were used to identify specific target genes for the candidate miRNAs.

qPCR analysis Selected candidate miRNAs (Table 1) were validated by quantitative real-time PCR (qPCR) as described by us previously $[15,18,19]$. Data are presented as DCT $\left(2^{-\Delta \mathrm{C}_{\mathrm{t}}}\right)$ expression.

Western blot analysis Total protein was extracted from the diabetic and non-diabetic mouse and human CPCs $(n=4-5$ in each group), separated with SDS-PAGE, transferred onto a PVDF membrane and probed with the following antibodies as detailed in the ESM Methods [15, 18, 19]: glycogen synthase kinase $3 \beta$ (GSK-3 beta, Novus Bio, NBP1-47470), myocytespecific enhancer factor 2C (MEF2C, Life Technologies, MA517119), cyclin-dependent kinase 6 (CDK6, Cell Signalling, 13331S), voltage-dependent anion-selective channel 1

Table 1 miRNAs with significant changes in diabetic mouse CPCs

\begin{tabular}{lcll}
\hline miRNA subtype & $\begin{array}{l}\text { Non-diabetic CPCs } \\
\text { (copy number) }\end{array}$ & $\begin{array}{l}\text { Diabetic CPCs } \\
\text { (copy number) }\end{array}$ & $p$ value \\
\hline miR-382-5p & $71.3 \pm 16.1$ & $170.9 \pm 12.2$ & 0.00117 \\
miR-376c-3p & $29.7 \pm 9.1$ & $115.8 \pm 20$ & 0.00446 \\
miR-329-3p & $51.6 \pm 19.3$ & $175 \pm 31.6$ & 0.01045 \\
miR-1983 & $43.3 \pm 8.4$ & $99.3 \pm 15$ & 0.01173 \\
miR-495-3p & $20.8 \pm 5.3$ & $48.3 \pm 7.3$ & 0.01614 \\
miR-28-5p & $129.6 \pm 19.4$ & $225.5 \pm 27.7$ & 0.02224 \\
miR-30c-5p & $478.1 \pm 122.6$ & $160.7 \pm 77.6$ & 0.00121 \\
miR-99b-5p & $174.2 \pm 12.6$ & $232.1 \pm 16.9$ & 0.02574 \\
miR-125b-3p & $68.4 \pm 12.3$ & $106.9 \pm 7.7$ & 0.02906 \\
miR-19a-3p & $155.8 \pm 42.5$ & $314.1 \pm 46.1$ & 0.03582 \\
miR-106b-5p & $106.1 \pm 15.5$ & $63.2 \pm 7.2$ & 0.03677 \\
miR-125a-5p & $2559 \pm 566$ & $4183 \pm 316$ & 0.03692 \\
miR-126-3p & $84.4 \pm 42.3$ & $407.6 \pm 125.4$ & 0.04062 \\
miR-804 & $98.9 \pm 7.5$ & $130.6 \pm 10.7$ & 0.04249 \\
miR-365-5p & $109 \pm 12.5$ & $151 \pm 12.5$ & 0.04479 \\
miR-132-3p & $194.5 \pm 24.9$ & $365.6 \pm 67.5$ & 0.04498 \\
\hline
\end{tabular}


(VDAC1, Abcam, ab15895), and $\beta$-actin (Novus Bio, NB-600503). Proteins were visualised using the Li-Cor Odyssey imaging station for fluorescence bands or by chemiluminescence reaction with enhanced chemiluminescence substrate (Pierce Fast Western Kit, SuperSignal West Pico). Target protein expression was normalised to either $\beta$-actin or total protein.

MS analysis Due to the difficulty in confirming the target genes predicted using the available online prediction tools (detailed in the Results section), we used MS to identify the potential miRNA targets as described earlier [30]. Two target genes, Vdacl and Cdk6, were selected as the most appropriate targets for the candidate miRNAs (detailed in the Results section).

Luciferase assay Mouse CPCs were co-transfected for $48 \mathrm{~h}$ with a pEZX-MT06 miR luciferase vector construct (GeneCopoeia) containing 3'-untranslated region (3'UTR) of wild-type Vdacl vector (identified as direct targets for miR$30 c-5 p$ by MS, explained in the Results section), mutant $V d a c 1$ vector, or wild-type and mutant $C d k 6$ vector (negative control to confirm there is no effect on the luciferase activity), and with miR-30c-5p mimics, or miRNA mimic scrambled controls, or with empty luciferase reporter vector. Luciferase activity was measured $48 \mathrm{~h}$ after transfection.

miR-30c overexpression and Vdac1 knockdown Human and mouse CPCs at passage four were transfected with miR-30c$5 \mathrm{p}$ mimic $(5 \mathrm{pmol} / \mu \mathrm{l})$ or miRNA mimic scrambled sequence $(5 \mathrm{pmol} / \mu \mathrm{l})$ or $V d a c l$ siRNA $(6 \mathrm{pmol} / \mu \mathrm{l})$ and negative control siRNA $(6 \mathrm{pmol} / \mu \mathrm{l})$ using lipofectamine RNAiMAX (ThermoFisher Scientific) according to the manufacturer's instructions. Transfection efficacy was confirmed by qPCR for miR-30c-5p and western blot analysis for VDAC1 expression.

Caspase-3/7 activity assay Diabetic and non-diabetic mouse and human CPCs at passage four were seeded at $1 \times 10^{4}$ cells/ well in a 96-well plate in a replicate of ten and transfected as above. Following $72 \mathrm{~h}$ transfection, caspase-3/7 activity was measured as described previously $[17,19]$.

Effect of miR-30c-5p on preserving mitochondrial membrane potential (MMP) Diabetic and non-diabetic mouse CPCs grown on glass coverslips $\left(1 \times 10^{4}\right.$ cells/coverslip $)$ or 96 well plates $\left(1 \times 10^{4}\right.$ cells/well $)$ were loaded with $1 \mu \mathrm{mol} / 1$ calcein-AM dye (Thermofisher Scientific) followed by treatment with $1 \mu \mathrm{mol} / 1 \mathrm{CoCl}_{2}$ to quench the calcein in the cytosol. After 15 min incubation at $37^{\circ} \mathrm{C}$, cells were either imaged using a fluorescent microscope or fluorescent intensity measured using a plate reader [31]

In vivo overexpression of miR-30c-5p in the diabetic heart To explore the functional role of miR-30c-5p in restoring the
CPC functions in vivo, aged (24-week-old) type 2 diabetic $(d b / d b)$ mice (representing the progressed stage of diabetes) received an injection of either miR-30c-5p mimic or a scrambled control $(10 \mathrm{nmol} / \mathrm{kg}$ in $0.2 \mathrm{ml}$ saline $[154 \mathrm{mmol} / 1 \mathrm{NaCl}]$, s.c., Exiqon, USA) after baseline echocardiography to confirm the existence of cardiac dysfunction. Animals were randomised by generating a single sequence of random values and matching them to one of the two treatment groups. The aged $d b / d b$ mice with cardiac dysfunction were used for this study to allow us to translate our findings to the clinic, because the majority of the patients attending the clinic have already developed some form of cardiac dysfunction. A blood sample and cardiac tissue were retrieved at the end of the 6 week follow-up period after echocardiography. Blood samples were used to measure the level of circulating miR-30c-5p. CPCs were isolated and characterised by flow cytometry as above. The effect of miR-30c-5p on VDAC1 expression was examined by western blot analysis, and improved survival was determined by CyQUANT analysis for cell proliferation and casapase-3/7 activity assay for apoptotic cell death.

Statistics All data are represented as mean \pm SEM. Normality of the data was confirmed using a Shapiro-Wilk test. A false discovery rate (using the Benjamini, Krieger and Yekitueli method) of 5\% was used to identify the significantly different miRNAs between diabetic and non-diabetic CPCs. A Student's $t$ test was used to compare the statistical differences between two groups, and all comparisons between multiple groups were obtained through a one-way or two-way ANOVA, with a Bonferroni post hoc test, or the KruskalWallis test, as appropriate. A $p$ value of less than 0.05 was considered significant. All data were analysed using GraphPad Prism 8.0 (USA).

\section{Results}

Diabetes-dysregulated miRNAs in mouse CPCs predicted to regulate their function Among 599 miRNAs evaluated in the miRNA expression profiling study, 16 were significantly different in diabetic CPCs (Table 1). The complete set of Nanostring data is presented in ESM Table 2. MiRPath software predicted that seven out of 16 significantly altered miRNAs to regulate signalling pathways associated with proliferation and self-renewal pathways (Table 2). Importantly, the effect of seven miRNAs on proliferation correlated with their altered expression profiles in the diabetic CPCs, with the miRNAs predicted to inhibit proliferation (miR-376c-3p, -329-3p, -495-3p, -19a-3p, -804 and -365-5p) being upregulated. In contrast, the only miRNA that was predicted to promote proliferation (miR-30c-5p) was downregulated in the diabetic CPCs (Tables 1 and 2). Since proliferation and self-renewal are the main properties of stem cells, 
Table 2 Predicted effect of miRNAs on cell proliferation

\begin{tabular}{lll}
\hline Signalling pathway & $\begin{array}{l}\text { miRNAs promoting } \\
\text { cell proliferation }\end{array}$ & miRNAs inhibiting cell proliferation \\
\hline Wnt & & miR-376c-3p \\
VEGF & miR-329-3p, -495-3p, -19a-3p \\
Hedgehog & & miR-376c-3p, -19a-3p, -365-5p \\
JAK-STAT & miR-30c-5p & miR-329-3p, -495-3p, -19a-3p, -365-5p \\
Focal adhesion & miR-30c-5p & miR-365-5p, miR-804 \\
PI3K-Akt & & miR-376c-3p, miR-365-5p \\
T2DM & & miR-329-3p, -495-3p, -19a-3p \\
\hline
\end{tabular}

VEGF, vascular endothelial growth factor; JAK, Janus kinases; STAT, signal transducer and activator of transcription proteins; PI3K, phosphoinositide 3-kinase; T2DM, type 2 diabetes mellitus seven predicted miRNAs possibly regulating CPC functions were chosen for further validation by qPCR analysis.

qPCR analysis partially validated the Nanostring mouse CPC data Interestingly, qPCR confirmed the changes in expression for only four miRNAs (miR-495-3p, miR329-3p, miR-376c-3p and miR-30c-5p [Fig. 1]). With their dysregulated expression validated by $q P C R$, these miRNAs were thus chosen as candidate miRNAs to determine target proteins responsible for the functional implications of the altered miRNAs.

\section{New targets identified in mouse CPCs using MS and validated} by western blot analysis Based on our initial analysis, $G s k 3 \beta$ and $M e f 2 c$ genes were predicted as a target for all four miRNAs, while Mef $2 c$ was predicted as a target for miR-329-3p, -376c-3p and -495-3p. GSK-3 $\beta$ and MEF2C regulate signalling pathways associated with cell proliferation and cell progression (ESM Fig. 2) [32, 33]. However, in contrast to our predictions, western blot analyses failed to show any change in the expression pattern

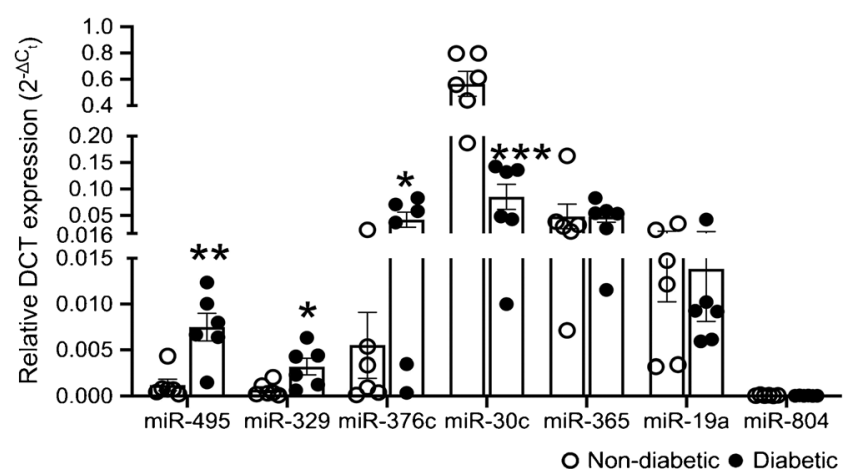

Fig. 1 qPCR validation of candidate miRNAs in mouse CPCs. Quantitative scatter plot bar graph showing the validation of candidate miRNAs identified by Nanostring analysis in diabetic and non-diabetic mouse CPCs. All the data are presented as mean \pm SEM; $n=6$ in each group. $* p<0.05, * * p<0.01$ and $* * * p<0.001$ vs non-diabetic CPCs in the corresponding group of either GSK-3 $\beta$ (ESM Fig. 3a) or MEF2C (ESM Fig. $3 b)$ in the diabetic CPCs.

We therefore used MS to identify new targets for the candidate miRNAs. Among the 2000 peptides evaluated by MS, 435 (22\%) were significantly dysregulated in diabetic CPCs. Among them, ten significantly dysregulated peptides (8 downregulated, two upregulated) constituted proteins that were predicted as targets for the candidate miRNAs (Table 3 and ESM Table 3). The cell cycle promoter CDK6 [34] was predicted to be a target for miR-329-3p, -495-3p and -376c-3p in four databases combined (miRDB, microT-CDS, microRNA.org and TargetScan) (Table 3). Western blot analysis confirmed significant downregulation of CDK6 in the diabetic CPCs ( $p=0.04$, Fig. 2a and ESM Fig. 4a).

The proapoptotic VDAC1 [35] was predicted as a target for miR-30c-5p in three databases (microT-CDS, TargetScan and microRNA.org) (Table 3). Western blot analysis confirmed significant upregulation of VDAC1 in the diabetic CPCs ( $p<0.01$, Fig. 2b and ESM Fig. 4b). CDK6 has been well characterised as playing a crucial role in stem cell proliferation [34]. However, to our knowledge, no study has demonstrated the role of VDAC1 in CPCs. Similarly, the role of miR-30c-5p in $\mathrm{CPCs}$ is not known. For these reasons, miR-30c-5p and its predicted target VDAC1 was selected for functional validation.

Luciferase assay confirmed Vdac1 as the direct target of miR30c-5p in mouse CPCs Transfection of mouse CPCs with miR$30 c-5 p$ mimic reduced the luminescence caused by the wildtype Vdac1-construct by $45 \pm 9 \%(p<0.01$, Fig. 2c), confirming that VDAC1 is the direct target for miR-30c-5p. There was no change in luminescence in mouse CPCs transfected with the mutant $V d a c l$ (Fig. 3c). Transfection with mutant and wild-type $C d k 6$-construct did not show any change in luminescence, validating the predicted results that CDK6 is not a direct target for miR-30c-5p (Fig. 2c).

Overexpression of miR-30c-5p reduced apoptotic cell death in diabetic mouse CPCs through downregulation of VDAC1 VDAC1 plays a major role in mitochondria-mediated 
Table 3 Predicted targets of candidate miRNA by MS

\begin{tabular}{|c|c|c|c|}
\hline Target proteins & $\begin{array}{l}\text { Fold change } \\
\text { (diabetic vs non-diabetic CPCs) }\end{array}$ & Candidate miRNAs predicted & Number of prediction databases \\
\hline \multicolumn{4}{|c|}{ Downregulated protein targets } \\
\hline KPNB1 & $0.39(p<0.001)$ & $\operatorname{miR}-376 c-3 p$ & 1 (TargetScan) \\
\hline \multirow[t]{3}{*}{ CDK6 } & $0.44(p<0.001)$ & $\operatorname{miR}-329-3 p$ & 2 (miRDB, RNA22) \\
\hline & & $\operatorname{miR}-495-3 p$ & 3 (TargetScan, miRDB, miRanda) \\
\hline & & $\operatorname{miR}-376 c-3 p$ & 1 (TargetScan) \\
\hline \multirow[t]{3}{*}{ GCC2 } & $0.54(p<0.001)$ & miR-329-3p & 1 (TargetScan) \\
\hline & & miR-495-3p & 1 (TargetScan) \\
\hline & & miR-376c-3p & 1 (TargetScan) \\
\hline \multirow[t]{2}{*}{ SARNP } & $0.60(p<0.01)$ & $\operatorname{miR}-329-3 p$ & 1 (TargetScan) \\
\hline & & miR-376c-3p & 1 (TargetScan) \\
\hline \multirow[t]{2}{*}{ GUCY1B1 } & $0.63(p=0.01)$ & miR-329-3p & 1 (TargetScan) \\
\hline & & $\operatorname{miR}-495-3 p$ & 2 (TargetScan, miRanda) \\
\hline \multirow[t]{2}{*}{ DNAJB4 } & $0.68(p=0.04)$ & miR-329-3p & 2 (TargetScan, miRanda) \\
\hline & & $\operatorname{miR}-30 c-5 p$ & 2 (miRanda, microRNA.org) \\
\hline \multirow[t]{2}{*}{ TPT1 } & $0.75(p<0.001)$ & miR-329-3p & 1 (TargetScan) \\
\hline & & $\operatorname{miR}-495-3 p$ & 1 (TargetScan) \\
\hline \multirow[t]{2}{*}{ CMPK1 } & $0.78(p<0.001)$ & $\operatorname{miR}-495-3 p$ & 2 (TargetScan, miRanda) \\
\hline & & $\operatorname{miR}-376 c-3 p$ & 1 (TargetScan) \\
\hline \multicolumn{4}{|c|}{ Upregulated protein targets } \\
\hline \multirow[t]{2}{*}{ MBNL2 } & $1.69(p=0.02)$ & $\operatorname{miR}-495-3 p$ & 1 (miRanda) \\
\hline & & $\operatorname{miR}-30 c-5 p$ & 1 (miRDB) \\
\hline VDAC1 & $1.67(p<0.01)$ & $\operatorname{miR}-30 c-5 p$ & 3 (RNA22, Target scan, microRN \\
\hline
\end{tabular}

KPNB1, karyopherin (importin) beta 1; GCC2, GRIP and coiled-coil domain containing 2; SARNP, SAP domain containing ribonucleoprotein; GUCY1B1, guanylate cyclase 1 soluble subunit $\beta$ 1; DNAJB4, DnaJ heat shock protein family (Hsp40) member B4; TPT1, tumour protein, translationally-controlled 1; CMPK1, cytidine/uridine monophosphate kinase 1; MBNL2, muscleblind like splicing regulator 2

apoptotic cell death [35], and diabetes accelerates apoptosis in stem cells $[19,36]$. In accordance with this, transfection of miR-30c-5p in both diabetic and non-diabetic CPCs (Fig. 3a) induced significant downregulation of VDAC1 protein in diabetic $(0.43 \pm 0.04$ arbitrary units [A.U.], $p=0.03)$ and non-diabetic CPCs $(0.33 \pm 0.04$ A.U., $p=0.002)$ compared with the scramble-treated control CPCs $(1.91 \pm 0.27$ A.U. in diabetic vs $0.58 \pm 0.04$ A.U. in non-diabetic CPCs) (Fig. 3b and ESM Fig. 5). Further, overexpression of miR-30c-5p resulted in $40 \pm 8 \%$ reduction in caspase- $3 / 7$ activity in the diabetic CPCs $(p<0.01$, Fig. $3 \mathrm{c})$, but not in the non-diabetic CPCs. Reduction in apoptosis was not associated with increased proliferation of cells as there was no difference in proliferation marker genes Mki67 and Top-2 $\alpha$ in any of the groups (Fig. 3d).

miR-30c-5p rescued MMP in diabetic mouse CPCs Our next aim was to determine whether overexpression of miR$30 \mathrm{c}-5 \mathrm{p}$ can preserve MMP, because VDAC1 allows the transit of metabolites across the mitochondrial outer membrane, thereby negatively regulating MMP [35].
Fluorescent images showed a significant reduction in calcein density in the diabetic CPCs after treatment with $\mathrm{CoCl}_{2}$, suggesting an open state or leaky mitochondrial permeability transition pore (PTP) in diabetic CPCs, allowing $\mathrm{CoCl}_{2}$ to quench calcein in the mitochondria, indicating reduced MMP (Fig. 3e, f). However, overexpression of mouse CPCs with miR-30c-5p restored the MMP in the diabetic CPCs (Fig. 3e, f). Further, knockdown of Vdacl recapitulated the prosurvival effects associated with miR-30c-5p overexpression, confirming VDAC1 as the underlying signalling mechanism for miR-30c-5p-associated beneficial effects (Fig. 4 and ESM Fig. 6).

In vivo overexpression of miR-30c-5p attenuated diabetesinduced deleterious effects on CPCs To determine whether the in vitro results can be reproduced in the in vivo setting, aged diabetic mice received injections of pre-miR-30c-5p mimic (Fig. 5a). qPCR analysis confirmed a significant increase in the expression of miR-30c-5p in both circulation and CPCs in mimic-treated animals (Fig. 5b, c). This was 
Fig. 2 Validation of targets predicted by MS of mouse CPCs. (a, b) Representative western blot images and quantitative scatter plot bar graphs showing the expression of CDK6 (a) and VDAC1 (b) in diabetic and nondiabetic mouse CPCs. Data are presented as the ratio of target protein and total protein and are mean $\pm \mathrm{SEM}, n=4$ in each group. $* p<0.05$ and $* * p<0.01$ vs nondiabetic. (c) Quantitative scatter plot bar graph showing luciferase activity of Vdacl- and Cdk6-3' UTR wild-type and mutant luciferase constructs expressed in mouse CPCs in the presence or absence of miR-30c-5pscrambled (Scr) or -mimic. Data are presented as relative luciferase activity normalised to wild-type Scr of Vdacl and Cdk6 separately and are mean \pm SEM, $n=4$ in each group. $* * * p<0.001$ vs the corresponding Scr-treated group
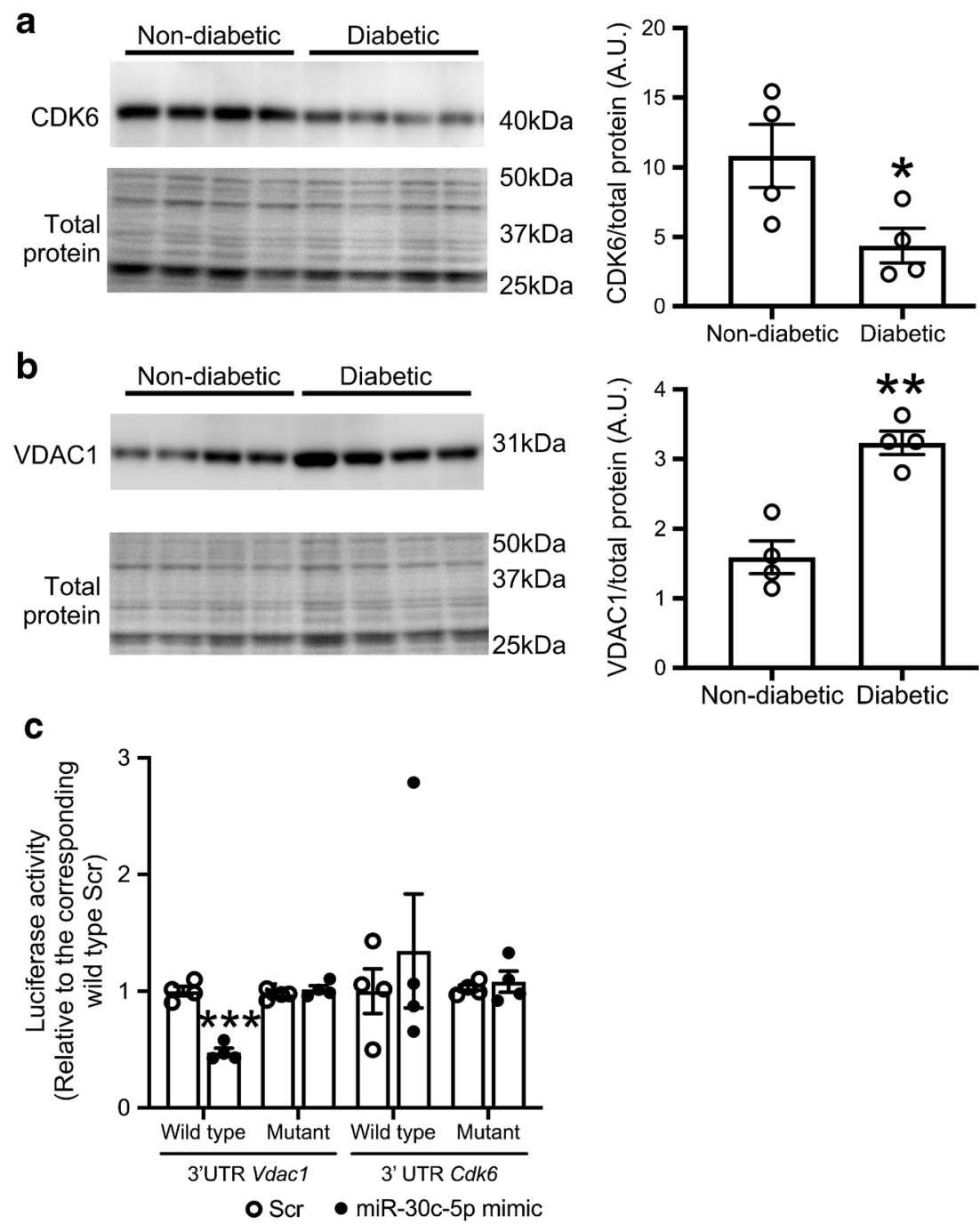

associated with marked downregulation of VDAC1 (Fig. 5d and ESM Fig. 7). Flow cytometry analysis showed a significant increase in the number of CPCs in the diabetic heart that were treated with mimic (Fig. 5e). CyQUANT analysis showed marked increase in the proliferation of CPCs (Fig. 5f), while caspase-3/7 assay demonstrated a significant reduction in the apoptotic cell death (Fig. 5g) in CPCs isolated from pre-miR-30c-5p-treated diabetic mice. Baseline echocardiography confirmed the existence of cardiac dysfunction in diabetic mice before the treatment (Table 4 and Fig. 5h, i). While there was no significant improvement in systolic function following treatment of diabetic mice with miR-30c-5p mimic, there was a marked improvement in diastolic function (E/A ratio, Table 4 and Fig. 5i).

\section{Diabetes induces dysregulation of target miRNAs in human} CPCs Finally, to determine if the results from mouse CPCs can be translated into the clinic, we performed a proof-ofconcept study on human CPCs. qPCR analysis confirmed the significant upregulation of miR-376c-3p and miR-495$3 \mathrm{p}(p<0.05$ for both) and downregulation of miR-30c-5p $(p<0.01)$, while miR-329-3p expression remained unaltered (Fig. 6a). Western blot analysis confirmed downregulation of CDK6 (Fig. 6b and ESM Fig. 8) and upregulation of VDAC1 in diabetic CPCs (Fig. 6c and ESM Fig. 9). Similar to the mouse CPCs, overexpression of miR-30c-5p significantly reduced the expression of VDAC1 (Fig. 6d and ESM Fig. 10) and caspase-3/7 activity (Fig. 6e) in diabetic human CPCs.

\section{Discussion}

To our knowledge, this is the first study to show the differential regulation of miRNAs associated with cell proliferation and survival in diabetic CPCs. Our in vitro and in vivo studies suggest that therapeutic modulation of miRNAs in diabetic CPCs can improve their functional efficacy.

For the current study, we used a heterogeneous cell population for both mouse and human heart. Sca- $1^{+}$cells represent 
a

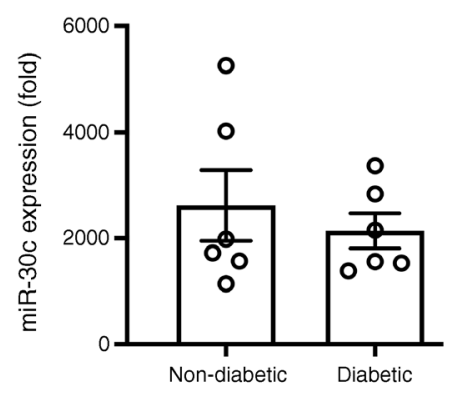

C

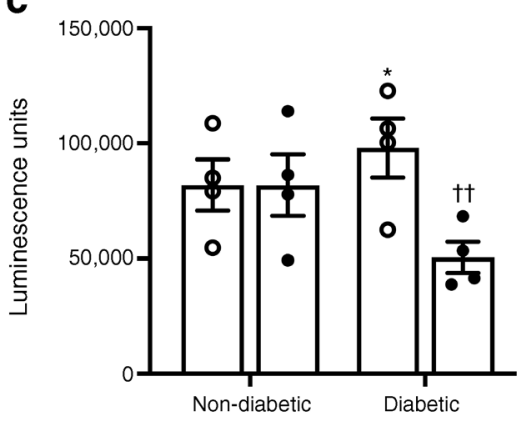

e

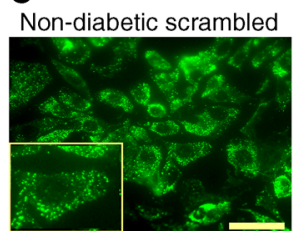

Diabetic scrambled

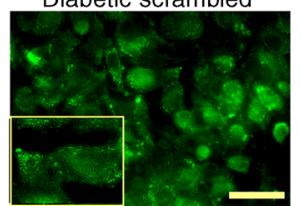

b
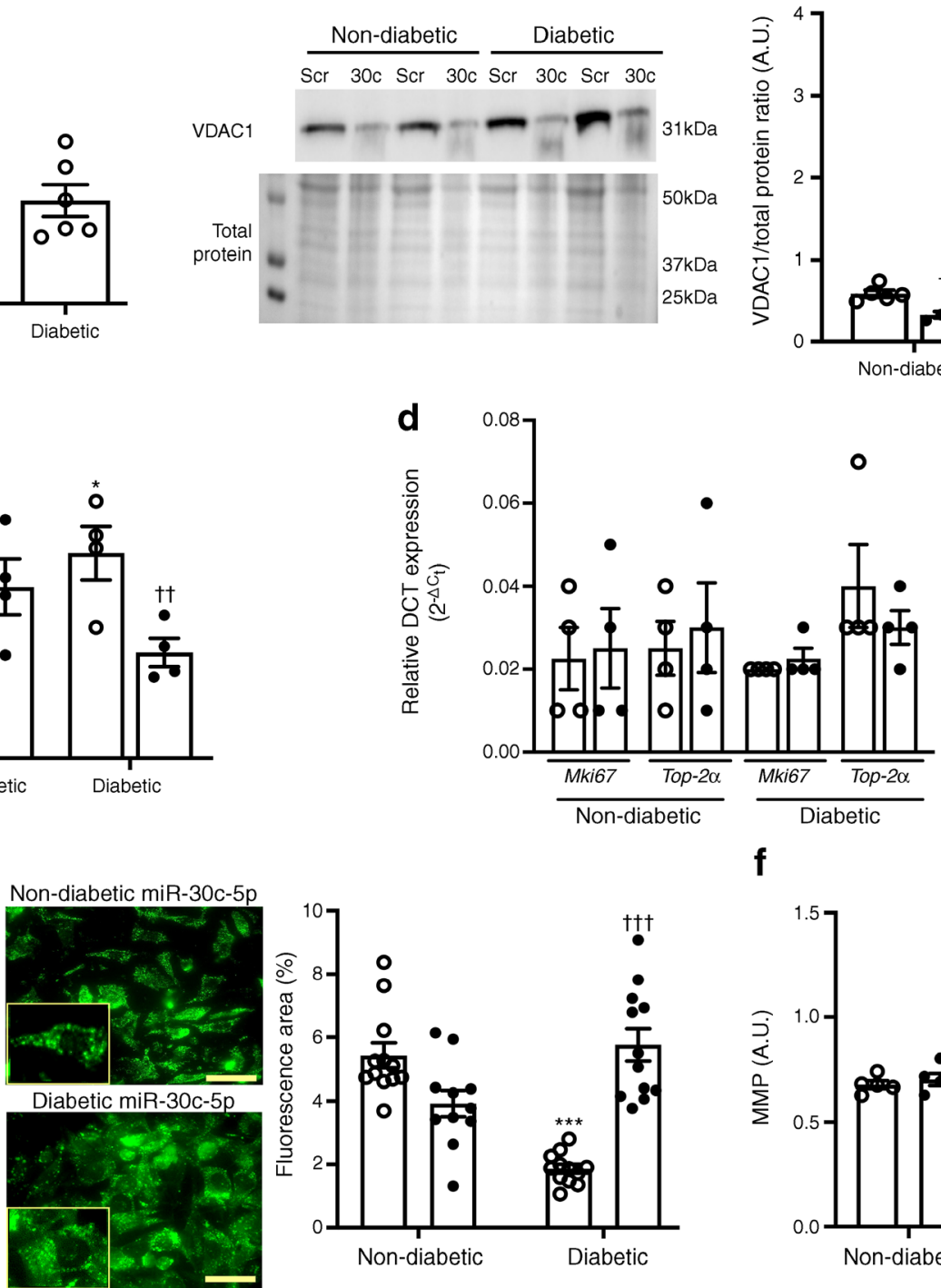
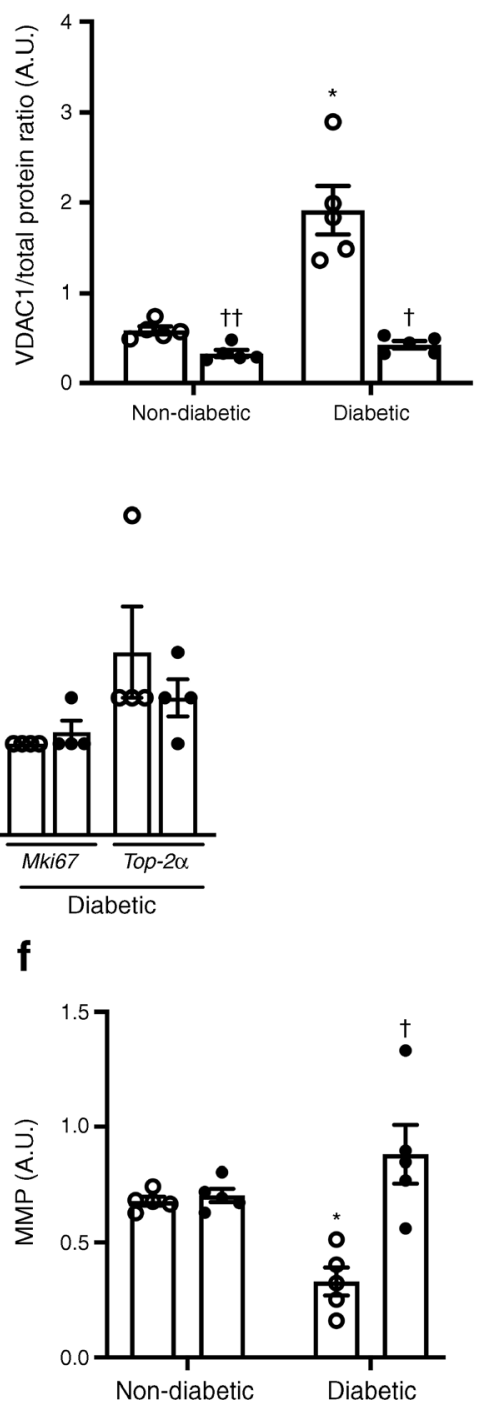

O scr
Fig. 3 Therapeutic modulation of diabetic mouse CPCs with miR-30c$5 \mathrm{p}$. CPCs isolated from non-diabetic and diabetic mice were transfected with scrambled (Scr) or miR-30c-5p mimic (30c). (a) Quantitative scatter plot bar graph showing a marked increase in the expression of miR-30c$5 \mathrm{p}$ in both diabetic and non-diabetic CPCs, $24 \mathrm{~h}$ after transfection. Data are presented as fold change compared with the Scr-treated group and $n=6$ in each group. (b) Representative western blot images and quantitative scatter plot bar graph showing VDAC1 protein expression in diabetic and non-diabetic CPCs after Scr or miR-30c-5p mimic treatment. Data are presented as the ratio of VDAC1 and total protein (A.U.) with $n=6$ in each group. (c, d) Quantitative scatter plot bar graphs exhibiting caspase-3/7 activity of transfected mouse CPCs (c) as well as mRNA

a heterogeneous population and possess the characteristics of CPCs, including the expression of cardiac-specific transcription factors as well as telomerase activity $[11,37,38]$. Either direct transplantation of Sca- $1^{+}$cells [39] or treatment with secretome released by the Sca- $1^{+}$cells [40] showed a significant improvement in cardiac function in a mouse model of expression of cell proliferation marker genes Mki67 and Top-2 $\alpha$ (d) in all the treated groups. Data are presented as luminescence units in (c) and relative DCT $\left(2^{-\Delta \mathrm{C}_{\mathrm{t}}}\right)$ expression in $(\mathbf{d}) ; n=4$ in each group. (e) Representative fluorescence microscopy images and quantitative scatter plot bar graph demonstrating calcein accumulation in cells as a measure of cell viability; $n=12$ in each group. Scale bars, $50 \mu \mathrm{m}$. (f) Quantitative scatter plot bar graph showing the MMP as a measurement of fluorescence intensity in all the study groups. Data are presented as A.U. with $n=5$ in each group. All the data are mean \pm SEM. $* p<0.05$, $*_{* *}^{*} p<0.001$ vs the corresponding non-diabetic group; ${ }^{\dagger} p<0.05$ and ${ }^{\dagger \dagger} p<0.001$ vs the corresponding Scr-treated group $2^{-\Delta \mathrm{C}_{\mathrm{t}}}$

myocardial infarction. Heterogenous human CPCs used in our study showed comparable characteristics to the Sca- $1^{+}$ population, expressing mesenchymal surface markers and CPC characteristics when cultured in vitro [23, 41]. In contrast to the other studies, we did not find any significant difference in the growth rate (proliferation) between diabetic and non- 
Fig. 4 Vdacl knockdown reduced apoptosis in diabetic mouse CPCs. (a) Quantitative scatter plot bar graph showing the expression pattern of $\mathrm{Vdacl}$ gene expression by qPCR; $n=4$ in each group. Data are presented as relative DCT $\left(2^{-\Delta C_{\mathrm{t}}}\right)$ expression. (b) Representative western blot images and quantitative scatter plot bar graph demonstrating fold difference in VDAC1 expression in diabetic and non-diabetic mouse CPCs after treatment with scrambled (Scr) or Vdacl siRNA (siRNA); $n=4$ in each group. Data are presented as the ratio of VDAC1 and total protein (A.U.). (c) Quantitative scatter plot bar graph showing caspase-3/7 activity in all the treated groups. Data are presented as luminescence units; $n=6$ in each group. All the data are mean \pm SEM. $* p<0.05, * * p<0.01$ and $* * * p<0.001$ vs the corresponding non-diabetic group; ${ }^{\dagger} p<0.05,{ }^{\dagger \dagger} p<0.01$ and ${ }^{\dagger \dagger} p<0.001$ vs the corresponding Scr-treated group a

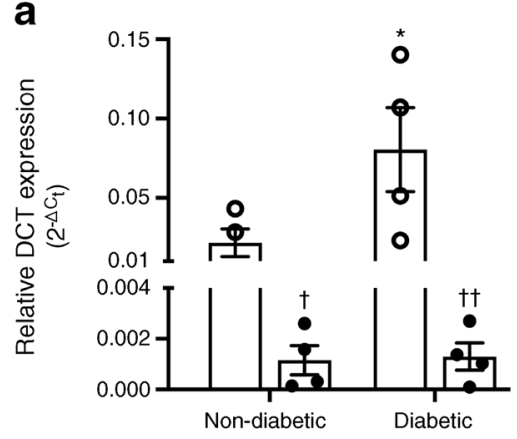

C

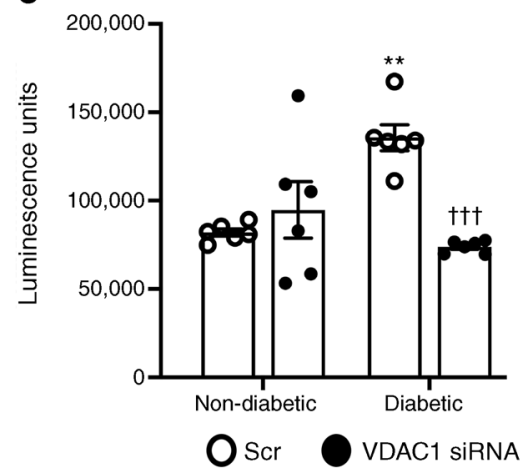

b
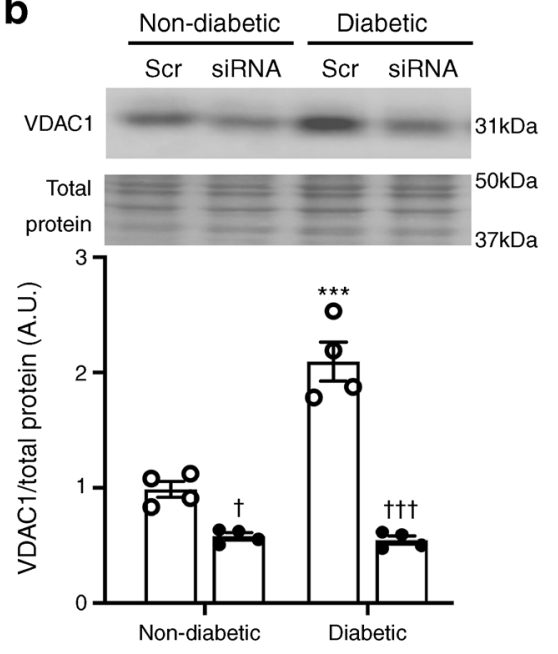

diabetic mouse CPCs in the CPCs isolated from young mice, which could be the reason for there being no difference in growth rate in the diabetic CPCs, although this was different in CPCs from the aged diabetic mice (discussed below). In support of this, our previous study showed reduced proliferation in the diabetic CPCs collected from 20-week-old diabetic mice [11]. The significant increase in apoptotic activity in diabetic human CPCs, which were collected from individuals with diabetes duration of 6 or more years, further confirms this notion. Therefore, the dysregulated miRNAs in our study are very likely to have functional effects at a later stage.

We focused on miRNAs associated with cell proliferation, differentiation and survival as these are the hallmarks of stem cells. Therefore, among the 16 miRNAs that were significantly altered in the diabetic mouse CPCs, we chose seven that are associated with cell survival, proliferation and differentiation. Interestingly, changes in miRNA expression could only be confirmed in four of the seven selected miRNAs (miR-329$3 p,-376 c-3 p,-495-3 p$ and $-30 c-5 p$ ), despite reports indicating superior sensitivity of Nanostring microarray over other techniques [42]. Studies on human CPCs demonstrated a similar dysregulation of three miRNAs (miR-30c-5p, -376c-3p and 329-3p) except in miR-329-3p, which remained unaltered in human diabetic CPCs. While the exact reason for this difference is not known, it is possible that all the diabetic patients were on treatment with either oral glucose-lowering agents or insulin, which could have either normalised or delayed the changes in miR-329-3p, although further studies are warranted.

Among the four miRNAs validated, miR-329 has been demonstrated to be associated with impaired angiogenesis, and knockdown of miR-329 increased endothelial cell proliferation [43]. In support of this observation, our bioinformatics analyses predicted that miR-329-3p inhibits cell proliferation (Table 2). The available evidence for the functional role of miR-376c-3p and miR-495-3p is limited. Overexpression of miR-376c inhibited the proliferation of osteosarcoma cells while inhibiting the differentiation of osteoblasts [44]. Similarly, increased expression of miR-495 inhibited proliferation of mesenchymal stem cells [45].

To date, there is no evidence for the expression of miR329-3p and -376c-3p and -495-3p in cardiomyocytes or CPCs. In support of this we also failed to detect the expression of these three miRNAs in normal human ventricular cardiomyocytes (ESM Fig. 11). However, marked upregulation of these miRNAs in the diabetic CPCs suggest that hyperglycaemic conditions could activate these miRNAs in the CPCs, thereby adversely affecting their functional efficacy. This was further supported by significant downregulation of the predicted target CDK6 in the diabetic CPCs. CDK6 is a $40 \mathrm{kDa}$ serine-threonine kinase that stimulates the G1/S phase of the cell cycle to promote cell proliferation. Studies have 
a

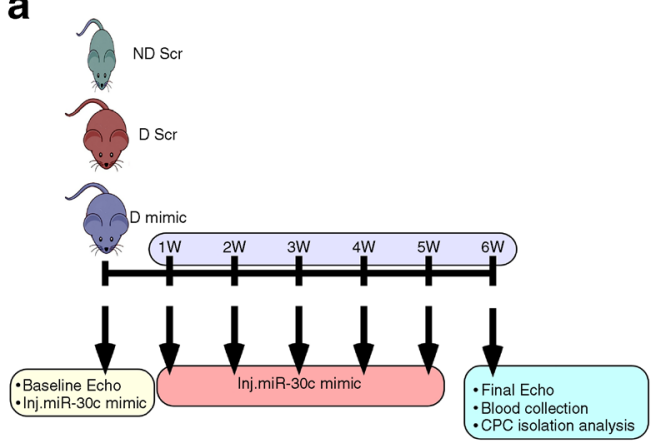

d
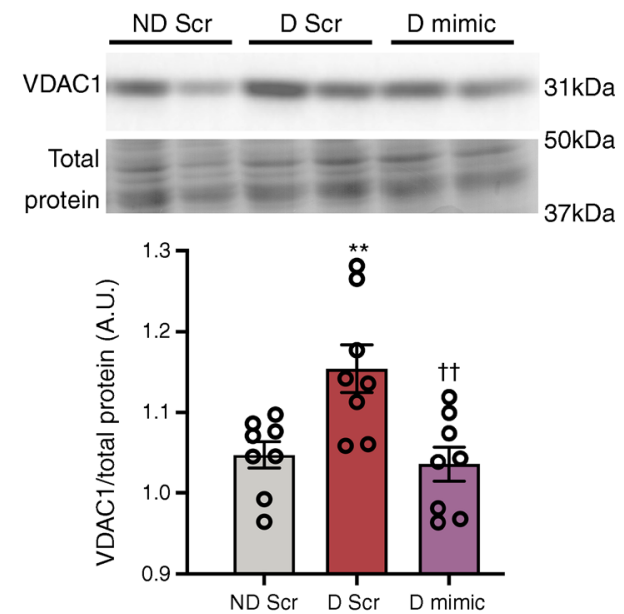

b

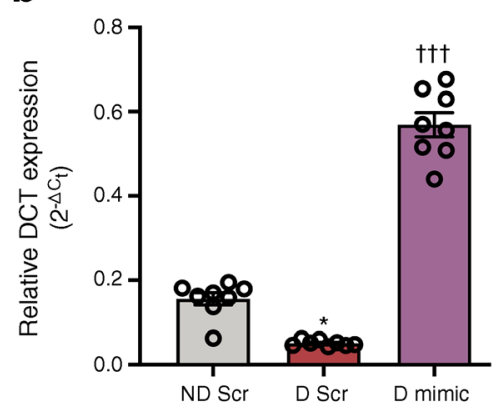

e
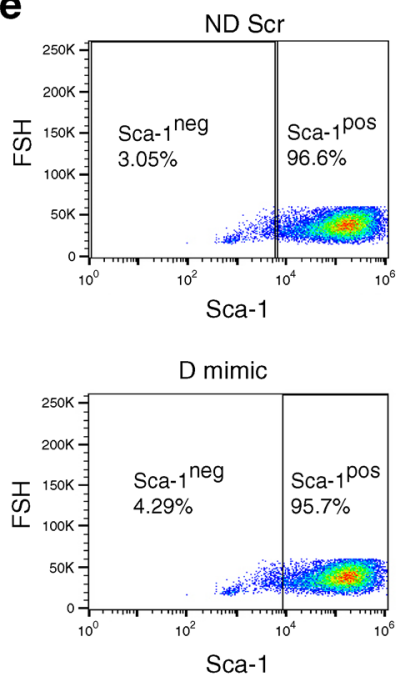

C

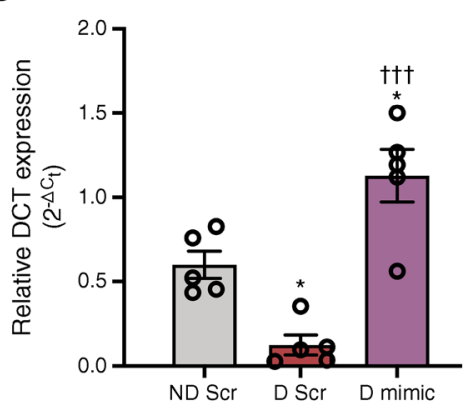

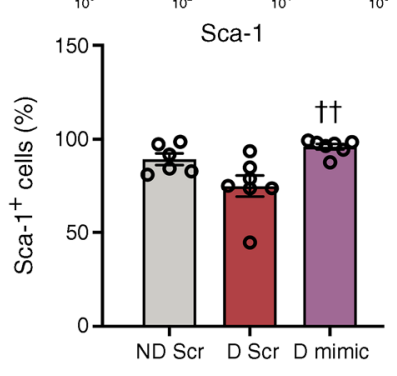

f

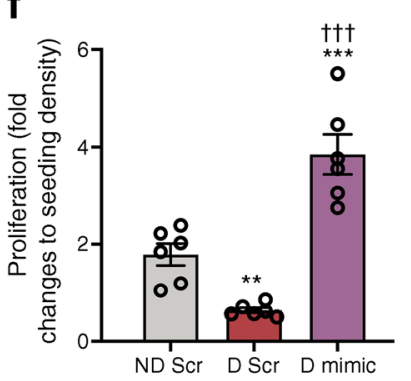

g

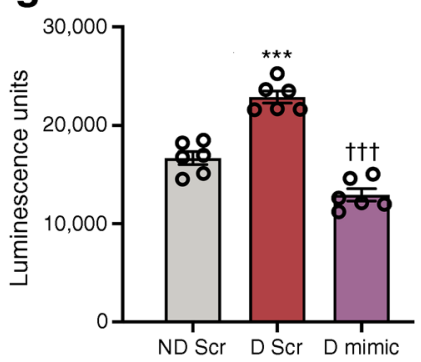

h

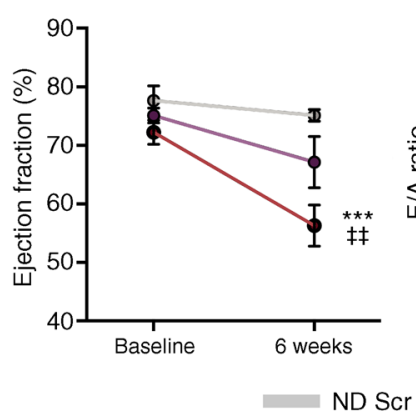

i

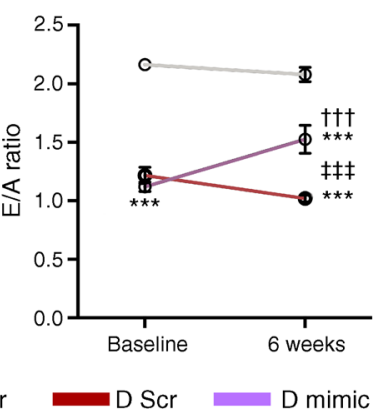

Fig. 5 In vivo overexpression of miR-30c-5p attenuated diabetesinduced deleterious effects on CPCs. (a) Experimental protocol used for in vivo experiments. W, week/weeks. (b, c) Quantitative scatter plot bar graphs showing the expression pattern of miR-30c-5p in circulation (b, $n=8$ in each group) and in CPCs (c, $n=5$ in each group). Data are presented as relative DCT $\left(2^{-\Delta C_{t}}\right)$ expression. (d) Western blot images and quantitative scatter plot bar graph demonstrating VDAC1 expression in diabetic and non-diabetic mouse CPCs after treatment with miR-30c$5 \mathrm{p}$ mimic or scramble (Scr); $n=8$ in each group. Data are presented as the ratio of VDAC1 and total protein (A.U.). (e) Flow cytometry scatter plot images and quantitative scatter plot bar graph showing the percentage of Sca- $1^{+}$cells in all the groups; $n=6$ in the non-diabetic Scr-treated group (ND Scr) and $n=7$ in the diabetic Scr (D Scr) and mimic (D mimic)

demonstrated the role of CDK6 in stem cell proliferation [34]. Knockdown of cyclin D2, an activator of CDK6, induces groups. (f, g) Quantitative scatter plot bar graphs showing the proliferation (f) and caspase-3/7 activity (g) in all the treated groups. Data are presented as fold change vs the initial seeding density (f) or luminescence units (g); $n=6$ in each group. (h, i) Quantitative line graphs showing ejection fraction (h) and E/A ratio (i) at baseline and after 6 weeks of treatment in each group; $n=8$ in each group. All data are presented as mean \pm SEM, (b-g) $* p<0.05, * * p<0.01$ and $* * * p<0.001$ vs ND Scrtreated group; ${ }^{\dagger \dagger} p<0.01$ and ${ }^{\dagger \dagger} p<0.001$ vs D Scr-treated group. (h, i) $* * * p<0.001$ vs ND Scr-treated group at corresponding time point; ${ }^{\dagger \dagger} p<0.001$ vs D Scr-treated group at corresponding time point; $\$ p<0.01$ vs baseline; $p=0.06$ for D Scr vs D mimic-treated group in (h). Echo, echocardiography; D, diabetic; mimic, miR-30c-5p mimic; ND, non-diabetic; Scr, scramble

insulin resistance and hence diabetes in the experimental animal model. Another study showed that prosenescent 
Table 4 Cardiac function at baseline and at 6 weeks after treatment

\begin{tabular}{|c|c|c|c|c|c|c|}
\hline \multirow[b]{2}{*}{ Variable } & \multicolumn{3}{|l|}{ Baseline } & \multicolumn{3}{|l|}{6 weeks } \\
\hline & ND Scr & D Scr & D mimic & ND Scr & D Scr & D mimic \\
\hline LVAWs (mm) & $1.45 \pm 0.03$ & $1.28 \pm 0.02 * *$ & $1.34 \pm 0.05^{*}$ & $1.51 \pm 0.03$ & $1.23 \pm 0.03 * * *$ & $1.33 \pm 0.05^{* *, \dagger}$ \\
\hline LVPWs (mm) & $1.26 \pm 0.05$ & $1.44 \pm 0.06$ & $1.32 \pm 0.05$ & $1.36 \pm 0.08$ & $1.31 \pm 0.03$ & $1.39 \pm 0.06$ \\
\hline LVPWd (mm) & $0.92 \pm 0.06$ & $0.98 \pm 0.04$ & $0.89 \pm 0.02$ & $0.95 \pm 0.05$ & $0.86 \pm 0.04$ & $1.00 \pm 0.03$ \\
\hline LVIDs (mm) & $1.90 \pm 0.1$ & $2.65 \pm 0.17 * * *$ & $2.4 \pm 0.10^{* *}$ & $2.08 \pm 0.05$ & $3.09 \pm 0.17 * * *,+$ & $2.9 \pm 0.23 * *,+t$ \\
\hline LVIDd (mm) & $3.34 \pm 0.04$ & $3.82 \pm 0.58$ & $3.62 \pm 0.08$ & $3.33 \pm 0.05$ & $4.13 \pm 0.04 * * *$ & $3.92 \pm 0.22 * *$ \\
\hline FS $(\%)$ & $43.5 \pm 2.8$ & $33 \pm 4.8 * * *$ & $32.2 \pm 2.9 * *$ & $38.2 \pm 1.7$ & $24.8 \pm 3.9 * *$ & $28.9 \pm 4.7^{*}$ \\
\hline $\operatorname{ESV}(\mu \mathrm{l})$ & $13.2 \pm 1.6$ & $18.1 \pm 1.5$ & $17.5 \pm 0.8$ & $15.6 \pm 0.7$ & $32.1 \pm 2.7 * * *$ & $23.8 \pm 3.3^{*, \dagger}$ \\
\hline $\operatorname{EDV}(\mu \mathrm{l})$ & $61.3 \pm 2.2$ & $69 \pm 2.0^{*}$ & $68.6 \pm 2.5^{*}$ & $62.8 \pm 2.2$ & $73.5 \pm 2.3 * *$ & $65.1 \pm 2.2$ \\
\hline $\mathrm{SV}(\mu \mathrm{l})$ & $48.1 \pm 1.7$ & $50.9 \pm 2.1$ & $51.1 \pm 2.5$ & $47.1 \pm 2.0$ & $41.5 \pm 3.1^{*}$ & $41.3 \pm 3.5^{\ddagger}$ \\
\hline $\mathrm{EF}(\%)$ & $78.9 \pm 2.5$ & $73.7 \pm 2.1$ & $74.4 \pm 1.3$ & $74.9 \pm 1.8$ & $56.2 \pm 3.6^{* * *, * ⿻ 一 未 丷 ~}$ & $63.9 \pm 4.4$ \\
\hline HR (bpm) & $502 \pm 5$ & $448 \pm 11 * *$ & $451 \pm 9 * *$ & $528 \pm 9$ & $461 \pm 11 * * *$ & $495 \pm 10^{\dagger \dagger}$ \\
\hline $\mathrm{CO}(\mathrm{ml})$ & $24.2 \pm 0.9$ & $22.8 \pm 0.7$ & $23.0 \pm 1.3$ & $24.9 \pm 1.2$ & $19.2 \pm 1.7 *$ & $20.4 \pm 1.8$ \\
\hline $\mathrm{E} / \mathrm{A}$ ratio & $2.2 \pm 0.03$ & $1.1 \pm 0.1 * * *$ & $1.1 \pm 0.04 * * *$ & $2.1 \pm 0.06$ & $0.99 \pm 0.03 * * *$ & $\begin{array}{l}1.5 \pm 0.12 \\
* * *,+\dagger, \mathrm{t}\end{array}$ \\
\hline
\end{tabular}

ND Scr, non-diabetic scramble; D Scr, diabetic scramble; D mimic, diabetic miR-30c-5p mimic; LVAWs, left ventricualr anterior wall at systole; LVPWs, left ventricular posterior wall at systole; LVPWd, LVPW at diastole; LVIDs, left ventricular internal diameter at systole; LVIDd, LVID at diastole; FS, fractional shortening; ESV, end systolic volume; EDV, end diastolic volume; SV, systolic volume; EF, ejection fraction; HR, heart rate; bpm, beats per min; CO, cardiac output. ${ }^{*} p<0.05,{ }^{*} p<0.01$ and $* * * p<0.001 \mathrm{vs} \mathrm{ND} \mathrm{Scr} \mathrm{at} \mathrm{the} \mathrm{corresponding} \mathrm{time} \mathrm{point;}{ }^{\dagger} p<0.05,{ }^{\dagger \dagger} p<0.01$ and ${ }^{+1} p<0.001$ D Scr vs D mimic of the corresponding time point; ${ }^{\star} p<0.05,{ }^{\mathrm{t}} p<0.01$ and ${ }^{\star \star} p<0.001$ baseline vs 6 weeks

miR-34a directly targets CDK6, and our previous study identified a marked upregulation of miR-34a in the diabetic heart $[19,46]$. Taken together, our results suggest that diabetesinduced activation of miR-329-3p, -376-3p and -495-3p in CPCs possibly inhibits their proliferation by downregulation of their target gene $C d k 6$.

miR-30c is one of the five members of the highly conserved miR-30 family (miR-30a-e) that share $85 \%$ sequence similarity. It is a mirtron derived from the miR$30 \mathrm{c}-1$ and miR-30c-2 genes that are encoded in intronic sequences in both humans and mice. Unlike the other three miRNAs, miR-30c has been demonstrated to be expressed by cardiomyocytes [47]. Studies have shown marked downregulation of miR-30c in the heart following myocardial infarction and ischaemia-reperfusion [47]. Importantly, miR-30c has been demonstrated to be downregulated in heart tissues collected from diabetic patients, which aligns with our current findings showing a marked downregulation of miR-30c in diabetic mouse and human CPCs [48]. Our study identified Vdacl as a new direct target for miR-30c-5p, and it was significantly upregulated in diabetic mouse and human CPCs. VDAC1 is a component of the mitochondrial PTP, the opening of which triggers apoptotic cell death. Opening of PTPs also allows for the diffusion of calcium ions into the mitochondria, causing calcium overload and accumulation of reactive oxygen species, leading to the loss of MMP [49]. This was true in our study showing impaired MMP in the diabetic mouse CPCs. Importantly, downregulation of VDAC1 and preserved MMP by miR-30c-5p overexpression, together with recapitulation of the effect of miR-30c-5p on CPC survival by direct knockdown of $\mathrm{Vdacl}$, demonstrated the involvement of VDAC1 as the downstream mechanism for miR-30c-5pinduced protective effects.

In vivo treatment of diabetic mice with miR-30c-5p improved CPC proliferation and survival. This is interesting because, as discussed above, we did not observe any changes in proliferation (growth rate) in diabetic CPCs after in vitro overexpression of miR-30c-5p, which is likely due to the difference in the age of CPCs used in our experiments. In vitro studies used CPCs collected from 10-week-old mice that did not exhibit cardiac dysfunction, whereas in vivo studies were conducted in 24-week-old mice that already exhibited cardiac dysfunction. Aged animals were used for the in vivo study as the majority of the patients attending the diabetes/ endocrinology or cardiology clinic are in older age groups and exhibit some form of cardiac dysfunction. We did not observe a significant improvement to the impaired systolic function following treatment of diabetic mice with miR-30c-5p mimic. This is likely to be attributed to the short-term follow-up nature of our study. In addition to its expression in CPCs, miR-30c-5p was also demonstrated to be expressed in cardiomyocytes exhibiting anti-apoptotic effects [48]. Therefore, it is possible that the beneficial effects, especially those related to cardiac function that are observed following in vivo injection of miR-30c-5p mimic, could be through improvement in cardiomyocyte survival. Future studies are 
Fig. 6 Expression and modification of candidate miRNAs and target proteins in human diabetic CPCs. (a) Quantitative scatter plot bar graph showing the expression pattern of candidate miRNAs by qPCR; $n=$ 6 in each group. Data are presented as relative DCT $\left(2^{-\Delta \mathrm{C}_{\mathrm{t}}}\right)$ expression. (b, c). Representative western blot images and quantitative scatter plot bar graphs exhibiting CDK6 (b) and VDAC1 (c) protein expression in diabetic and non-diabetic human CPCs; $n=5$ in each group. Data are presented as the ratio of CDK6 (b) or VDAC1 (c) and total protein (A.U.). (d)

Representative western blot images and quantitative scatter plot bar graph demonstrating VDAC1 expression in diabetic and non-diabetic human CPCs after treatment with scrambled (Scr) or miR-30c-5p mimic; $n=6$ in each group. Data are presented as the ratio of VDAC1 and total protein (A.U.). (e) Quantitative scatter plot bar graph showing the caspase- $3 / 7$ activity in all the treated groups. Data are presented as luminescence units; $n=6$ in each group. All data are presented as mean \pm SEM $* p<0.05$, $* * p<0.01$ and $* * * p<0.001$ vs the corresponding non-diabetic group; ${ }^{\dagger} p<0.05$ and ${ }^{\dagger \dagger} p<0.01$ vs the corresponding Scr-treated group

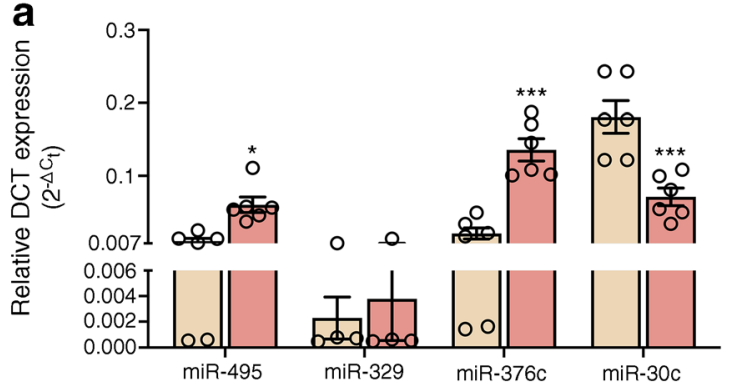

b

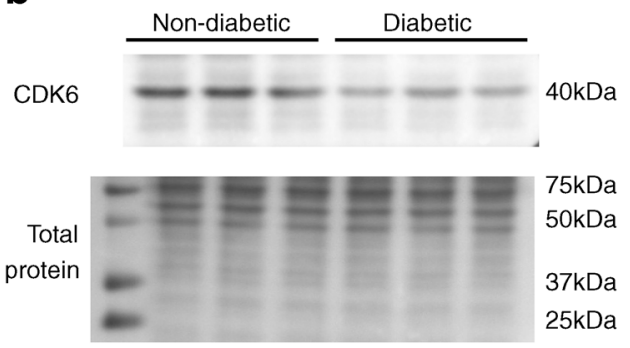

C
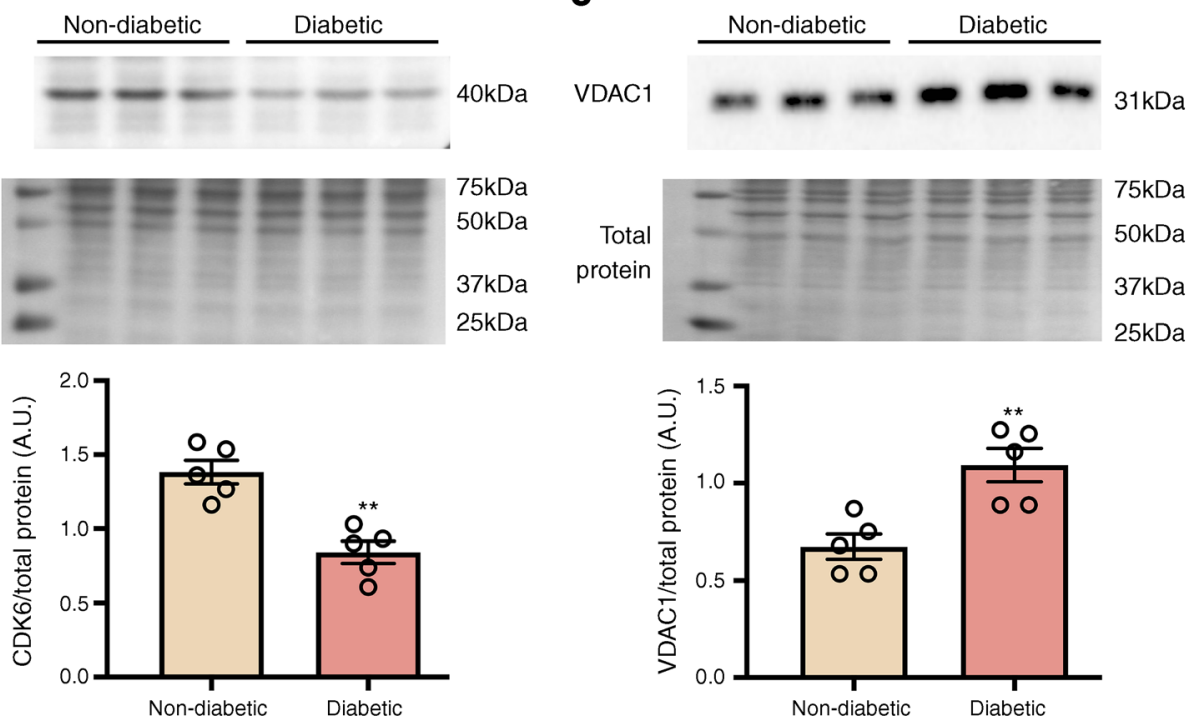

d

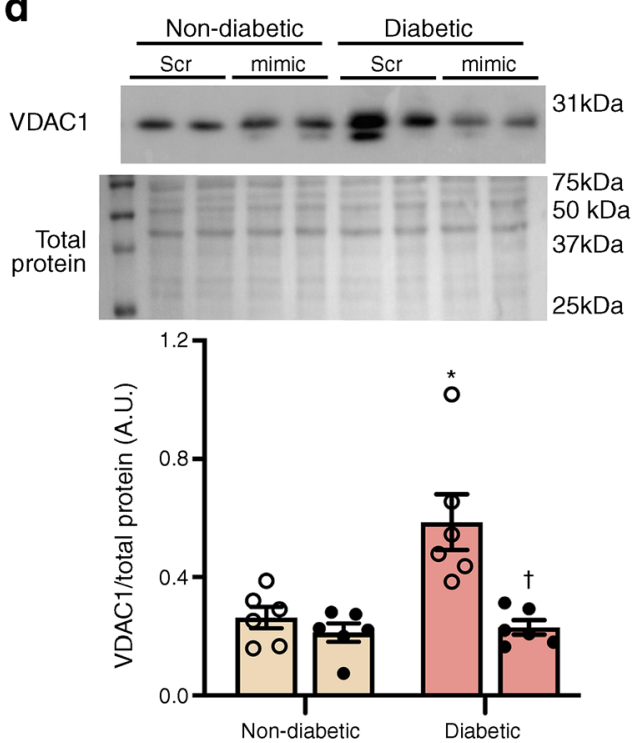

e

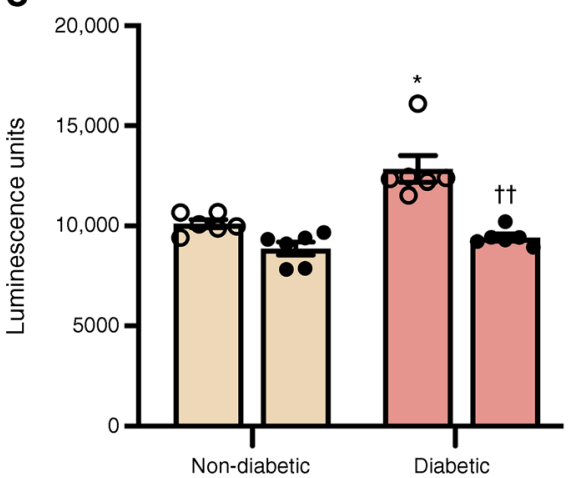

- Scr miR-30c-5p mimic required to determine the effect of mIR-30c-5p mimic treatment on other cell types in the heart.

Although our approach of delivering miR-30c-5p to the diabetic animals without specifically targeting CPCs did not indicate any noticable adverse effects, it is important to consider other possible modes of CPC-directed delivery of
miRNAs. This could be achieved by conjugating miRNAs with CPC-specific promoters or receptors to direct the delivery of miRNAs specifically to the CPCs.

In conclusion, our results have newly identified that diabetes induces dysregulation of three miRNAs (miR-30c-5p, -376c-3p and $-329-3 p$ ) that are associated with stem cell survival and 
proliferation. This was associated with marked downregulation of the cell proliferation activator CDK6 (target for miR-30c-5p, -329-3p and $-376 c-3 p$ ) and upregulation of proapoptotic VDAC1, which we identified as a new target for miR-30c-5p. Therapeutic modulation of mouse (in vitro and in vivo) and human (in vitro) diabetic CPCs with miR-30c-5p significantly reduced apoptotic cell death and loss of MMP. Moreover, protective effects of miR-30c-5p on human CPCs has provided a strong foundation for the need for larger clinical studies. Altogether, our results suggest that modification of miRNA expression could be an effective approach to improve the therapeutic potential of diabetic CPCs. However, further long-term in vivo studies are required as the next step towards translation of this new treatment into the clinic.

Supplementary Information The online version contains peer-reviewed but unedited supplementary material available at https://doi.org/10.1007/ s00125-021-05405-7.

Acknowledgements We thank the staff nurses at the surgical theatre for helping with human sample collection. We also thank the animal welfare technicians for their help in daily monitoring and care of our experimental animals.

Data availability The data that support the findings of this study are available within the article and supplementary files, or available from the corresponding authors upon request.

Funding This study was supported by research fundings from the Heart Foundation New Zealand (1600), Royal Society of New Zealand catalyst funding (18-UOO-011-CSG), Lottery Health research funding (R-LHR2020-128280), Department of Physiology (AIM Fund) and School of Biomedical Sciences Dean's Bequest funding. NP was supported by a University of Otago Doctoral Scholarship.

Authors' relationships and activities The authors declare that there are no relationships or activities that might bias, or be perceived to bias, their work.

Contribution statement All authors contributed to the interpretation of data and reviewing of the manuscript. NP performed all the mouse experimental work and data analysis, and drafted the manuscript; SK and SG performed human CPC experiments; DC and JBP conducted analysis following in vivo miR-30c experiments; IVH maintained the database for human tissue samples; SC and MJAW supervised echocardiography and analysis; RB, RS, DP and PD collected the human tissue samples; RK and $\mathrm{AB}$ conceived, designed and supervised the study, and wrote the final version of the manuscript. All the authors have approved the final version to be published. RK and $\mathrm{AB}$ are the guarantors of this work.

\section{References}

1. World Heart Federation (2020) The world's most common cause of death. Available from www.world-heart-federation.org/wpcontent/uploads/2017/05/WCC2016_CVDs_infographic-1.pdf. Accessed Feb 2020

2. Isomi M, Sadahiro T, Ieda M (2019) Progress and challenge of cardiac regeneration to treat heart failure. J Cardiol 73:97-101. https://doi.org/10.1016/j.jjcc.2018.10.002
3. Satthenapalli VR, Lamberts RR, Katare RG (2017) Concise review: Challenges in regenerating the diabetic heart: A comprehensive review. Stem Cells 35:2009-2026. https://doi.org/10.1002/stem.2661

4. Tzahor E, Poss KD (2017) Cardiac regeneration strategies: Staying young at heart. Science 356:1035-1039. https://doi.org/10.1126/ science.aam5894

5. The Lancet Editors (2014) Expression of concern: The SCIPIO trial. Lancet 383:1279. https://doi.org/10.1016/S0140-6736(14) 60608-5

6. The American Heart Association (2019) Retraction of: Cardiomyogenesis in the developing heart is regulated by C-kitpositive cardiac stem cells. Circ Res 124:e28. https://doi.org/10. 1161/RES.0000000000000252

7. The American Heart Association (2019) Retraction of: Role of cardiac stem cells in cardiac pathophysiology: A paradigm shift in human myocardial biology. Circ Res 124:e24. https://doi.org/10. 1161/RES.0000000000000248

8. Gude NA, Sussman MA (2019) Cardiac regenerative therapy: Many paths to repair. Trends Cardiovasc Med 30(6):338-343

9. Abbott JD, Huang Y, Liu D, Hickey R, Krause DS, Giordano FJ (2004) Stromal cell-derived factor-1alpha plays a critical role in stem cell recruitment to the heart after myocardial infarction but is not sufficient to induce homing in the absence of injury. Circulation 110:3300 3305. https://doi.org/10.1161/01.CIR.0000147780.30124.CF

10. Hatzistergos KE, Saur D, Seidler B et al (2016) Stimulatory effects of mesenchymal stem cells on cKit+ cardiac stem cells are mediated by SDF1/CXCR4 and SCF/cKit signaling pathways. Circ Res 119: 921-930. https://doi.org/10.1161/CIRCRESAHA.116.309281

11. Katare R, Oikawa A, Cesselli D et al (2013) Boosting the pentose phosphate pathway restores cardiac progenitor cell availability in diabetes. Cardiovasc Res 97:55-65. https://doi.org/10.1093/cvr/ cvs291

12. Khaksar M, Sayyari M, Rezaie J, Pouyafar A, Montazersaheb S, Rahbarghazi R (2018) High glucose condition limited the angiogenic/cardiogenic capacity of murine cardiac progenitor cells in in vitro and in vivo milieu. Cell Biochem Funct 36:346-356. https://doi.org/10.1002/cbf.3354

13. Mehra P, Guo Y, Nong Y et al (2018) Cardiac mesenchymal cells from diabetic mice are ineffective for cell therapy-mediated myocardial repair. Basic Res Cardiol 113:46

14. Palomer X, Pizarro-Delgado J, Vazquez-Carrera M (2018) Emerging actors in diabetic cardiomyopathy: Heartbreaker biomarkers or therapeutic targets? Trends Pharmacol Sci 39:452467. https://doi.org/10.1016/j.tips.2018.02.010

15. Rawal S, Nagesh PT, Coffey S et al (2019) Early dysregulation of cardiac-specific microRNA-208a is linked to maladaptive cardiac remodelling in diabetic myocardium. Cardiovasc Diabetol 18:13

16. Hathaway QA, Pinti MV, Durr AJ, Waris S, Shepherd DL, Hollander JM (2018) Regulating microRNA expression: At the heart of diabetes mellitus and the mitochondrion. Am J Physiol Heart Circ Physiol 314:H293-H310. https://doi.org/10.1152/ ajpheart.00520.2017

17. Rawal S, Munasinghe PE, Shindikar A et al (2017) Downregulation of proangiogenic microRNA-126 and microRNA-132 are early modulators of diabetic cardiac microangiopathy. Cardiovasc Res 113:90-101. https://doi.org/10.1093/cvr/cvw235

18. Rawal S, Munasinghe PE, Nagesh PT et al (2017) Down-regulation of miR-15a/b accelerates fibrotic remodelling in the type 2 diabetic human and mouse heart. Clin Sci (Lond) 131:847-863. https://doi. org/10.1042/CS20160916

19. Fomison-Nurse I, Saw EEL, Gandhi S et al (2018) Diabetes induces the activation of pro-ageing miR-34a in the heart, but has differential effects on cardiomyocytes and cardiac progenitor cells. Cell Death Differ 25:1336-1349. https://doi.org/10.1038/s41418-017-0047-6

20. Gorabi AM, Bianconi V, Pirro M, Banach M, Sahebkar A (2019) Regulation of cardiac stem cells by microRNAs: State-of-the-art. 
Biomed Pharmacother 120:109447. https://doi.org/10.1016/j. biopha.2019.109447

21. Chandrasekera DNK, Neale JPH, van Hout I et al (2020) Upregulation of microRNA-532 enhances cardiomyocyte apoptosis in the diabetic heart. Apoptosis 25:388-399. https://doi.org/10. 1007/s10495-020-01609-1

22. Lew JK, Pearson JT, Saw E et al (2020) Exercise regulates microRNAs to preserve coronary and cardiac function in the diabetic heart. Circ Res 127(11):1384-1400. https://doi.org/10. 1161/CIRCRESAHA.120.317604

23. Dixit P, Katare RG (2019) Isolation and characterization of cardiac progenitor cells. Methods Mol Biol 2029:161-173

24. Rawal S, Ram TP, Coffey S et al (2016) Differential expression pattern of cardiovascular microRNAs in the human type-2 diabetic heart with normal ejection fraction. Int J Cardiol 202:40-43. https:// doi.org/10.1016/j.ijcard.2015.08.161

25. Manning P, Munasinghe PE, Bellae Papannarao J, Gray AR, Sutherland W, Katare R (2019) Acute weight loss restores dysregulated circulating MicroRNAs in individuals who are obese. J Clin Endocrinol Metab 104:1239-1248. https://doi.org/10.1210/jc. 2018-00684

26. Paraskevopoulou MD, Georgakilas G, Kostoulas N et al (2013) DIANA-microT web server v5.0: Service integration into miRNA functional analysis workflows. Nucleic Acids Res 41:W169W173. https://doi.org/10.1093/nar/gkt393

27. Wong N, Wang X (2015) miRDB: An online resource for microRNA target prediction and functional annotations. Nucleic Acids Res 43:D146-D152. https://doi.org/10.1093/nar/gku1104

28. Betel D, Wilson M, Gabow A, Marks DS, Sander C (2008) The microRNA.Org resource: Targets and expression. Nucleic Acids Res 36:D149-D153. https://doi.org/10.1093/nar/gkm995

29. Agarwal V, Bell GW, Nam JW, Bartel DP (2015) Predicting effective microRNA target sites in mammalian mRNAs. Elife 4:e05005

30. Szklarczyk D, Morris JH, Cook H et al (2017) The STRING database in 2017: Quality-controlled protein-protein association networks, made broadly accessible. Nucleic Acids Res 45:D362 D368. https://doi.org/10.1093/nar/gkw937

31. Hom JR, Quintanilla RA, Hoffman DL et al (2011) The permeability transition pore controls cardiac mitochondrial maturation and myocyte differentiation. Dev Cell 21:469-478

32. Esfandiari F, Fathi A, Gourabi H, Kiani S, Nemati S, Baharvand H (2012) Glycogen synthase kinase-3 inhibition promotes proliferation and neuronal differentiation of human-induced pluripotent stem cell-derived neural progenitors. Stem Cells Dev 21:32333243. https://doi.org/10.1089/scd.2011.0678

33. Li H, Radford JC, Ragusa MJ et al (2008) Transcription factor MEF2C influences neural stem/progenitor cell differentiation and maturation in vivo. Proc Natl Acad Sci U S A 105:9397-9402

34. Scheicher R, Hoelbl-Kovacic A, Bellutti F et al (2015) CDK6 as a key regulator of hematopoietic and leukemic stem cell activation. Blood 125:90-101. https://doi.org/10.1182/blood-2014-06-584417

35. Shore GC (2009) Apoptosis: it's BAK to VDAC. EMBO Rep 10: 1311-1313. https://doi.org/10.1038/embor.2009.249

36. Rota M, LeCapitaine N, Hosoda $\mathrm{T}$ et al (2006) Diabetes promotes cardiac stem cell aging and heart failure, which are prevented by deletion of the p66shc gene. Circ Res 99:42-52. https://doi.org/10. 1161/01.RES.0000231289.63468.08

37. Noseda M, Harada M, McSweeney S et al (2015) PDGFRalpha demarcates the cardiogenic clonogenic Sca1+ stem/progenitor cell in adult murine myocardium. Nat Commun 6:6930
38. Katare R, Caporali A, Zentilin L et al (2011) Intravenous gene therapy with PIM-1 via a cardiotropic viral vector halts the progression of diabetic cardiomyopathy through promotion of prosurvival signaling. Circ Res 108:1238-1251. https://doi.org/10.1161/ CIRCRESAHA.110.239111

39. Marunouchi T, Sasaki K, Yano E, Tanonaka K (2019) Transplantation of cardiac Sca-1-positive cells rather than c-Kitpositive cells preserves mitochondrial oxygen consumption of the viable myocardium following myocardial infarction in rats. $\mathrm{J}$ Pharmacol Sci 140:236-241. https://doi.org/10.1016/j.jphs.2019. 07.005

40. Konemann S, Sartori LV, Gross S et al (2020) Cardioprotective effect of the secretome of Sca-1+ and Sca-1- cells in heart failure: Not equal, but equally important? Cardiovasc Res 116:566-575. https://doi.org/10.1093/cvr/cvz140

41. Dixit P, Donnelly H, Edamatsu M, Galvin I, Bunton R, Katare R (2017) Progenitor cells from atria, ventricle and peripheral blood of the same patients exhibit functional differences associated with cardiac repair. Int J Cardiol 228:412-421. https://doi.org/10.1016/ j.ijcard.2016.11.178

42. Veldman-Jones MH, Brant R, Rooney C et al (2015) Evaluating robustness and sensitivity of the NanoString technologies nCounter platform to enable multiplexed gene expression analysis of clinical samples. Cancer Res 75:2587-2593. https://doi.org/10.1158/00085472.CAN-15-0262

43. Welten SM, Bastiaansen AJ, de Jong RC et al (2014) Inhibition of 14q32 MicroRNAs miR-329, miR-487b, miR-494, and miR-495 increases neovascularization and blood flow recovery after ischemia. Circ Res 115:696-708. https://doi.org/10.1161/ CIRCRESAHA.114.304747

44. Kureel J, John AA, Prakash R, Singh D (2018) MiR 376c inhibits osteoblastogenesis by targeting Wnt3 and ARF-GEF-1 -facilitated augmentation of beta-catenin transactivation. J Cell Biochem 119: 3293-3303. https://doi.org/10.1002/jcb.26490

45. Lee S, Yoon DS, Paik S, Lee KM, Jang Y, Lee JW (2014) microRNA-495 inhibits chondrogenic differentiation in human mesenchymal stem cells by targeting Sox 9 . Stem Cells Dev 23: 1798-1808. https://doi.org/10.1089/scd.2013.0609

46. Sun F, Fu H, Liu Q et al (2008) Downregulation of CCND1 and CDK6 by miR-34a induces cell cycle arrest. FEBS Lett 582:1564 1568. https://doi.org/10.1016/j.febslet.2008.03.057

47. Duisters RF, Tijsen AJ, Schroen B et al (2009) miR-133 and miR30 regulate connective tissue growth factor: Implications for a role of microRNAs in myocardial matrix remodeling. Circ Res 104: 170-178, 176p following 178. https://doi.org/10.1161/ CIRCRESAHA.108.182535

48. Raut SK, Singh GB, Rastogi B et al (2016) miR-30c and miR-181a synergistically modulate p53-p21 pathway in diabetes induced cardiac hypertrophy. Mol Cell Biochem 417:191-203. https://doi. org/10.1007/s11010-016-2729-7

49. Naghdi S, Hajnoczky G (2016) VDAC2-specific cellular functions and the underlying structure. Biochim Biophys Acta 1863:25032514

Publisher's Note Springer Nature remains neutral with regard to jurisdictional claims in published maps and institutional affiliations. 